\title{
Integrating canopy and large-scale effects in the convective boundary-layer dynamics during the CHATS experiment
}

Metodija M. Shapkalijevski et al.

Correspondence to: Metodija M. Shapkalijevski (metoshapkalijevski@gmail.com)

The copyright of individual parts of the supplement might differ from the CC-BY 3.0 licence. 
To calculate the boundary-layer height observed during the CHATS experiment (Patton et al., 2011), we make use of the NCAR Raman-shifted Eye-Safe Aerosol Lidar (REAL) backscatter signal data. The application of REAL at CHATS experiment, its purpose and operational characteristics in the campaign are presented in Patton et al. (2011). Here we describe the algorithm employed to calculate the boundary-layer height using the above mentioned data.

The automatic method to calculate the boundary-layer height, which is presented in this document, is based on the method of the height of the maximal vertical gradient of the aerosol backscatter profiles (Endlich et al., 1979). The steps of the algorithm are presented below.

1) Visualisation of the two-dimensional (elevation-height) backscatter data

The data is stored as two-dimensional arrays (distance from the LIDAR, elevation). Each of the 175 arrays (175 elevations) for certain time-period (around 11 seconds) has 4200 signal cells with equidistant spacing of 1.5 meter. To construct profiles of the signal in height and distance from the LIDAR, the data is transferred from polar coordinates to Cartesian coordinate system (e.g. Fig. S1).

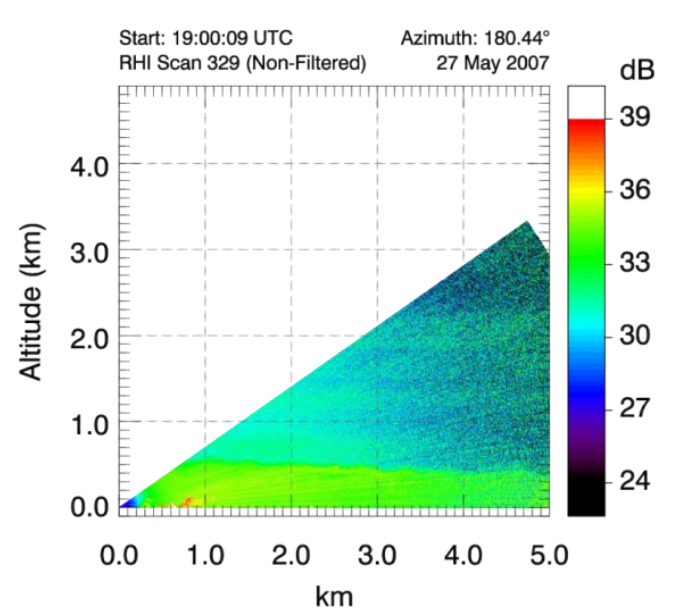

Figure S1: REAL backscatter data (in dB) on 27 May 2007 at 19:00:09 UTC (12:00:09 LT), plotted in height (y-axis) and distance from the LIDAR (x-axis), and for several elevations.

The aerosol backscatter shows the aerosol concentration $(\mathrm{dB})$ in height and distance from the lidar. The maximum gradient of the aerosol concentration marks the interface between the atmospheric boundary layer and the free troposphere (in this particular example around $500 \mathrm{~m}$, Fig. S1). In the following paragraphs, we explain the criteria used to find this maximum aerosol gradient.

2) Retrieving the backscatter vertical profiles

Two methods of retrieving the profiles from the raw backscatter data are used and compared: i) using a single profile at 50 per cent of the distance (i.e. at $2500 \mathrm{~m}$ ), or ii) calculating an average profile from the profiles within a certain range. We averaged all the profiles between the $1000^{\text {th }}$ and $2000^{\text {th }}$ cell (in total 1000 profiles). Figure S2 shows a typical example using both methods. 


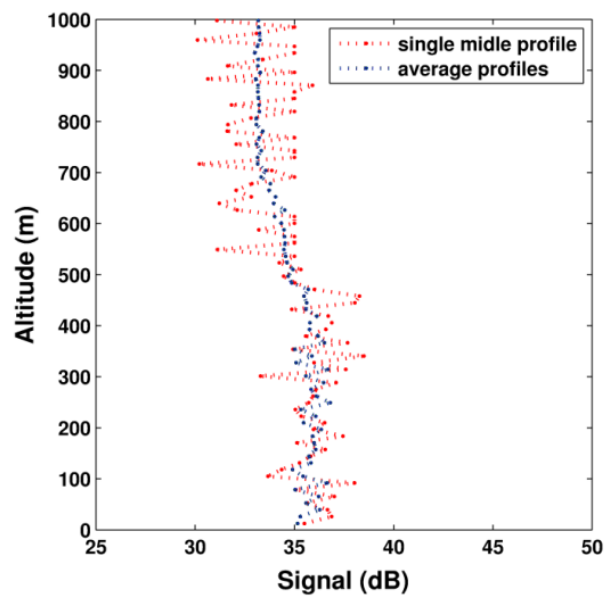

Figure S2: Retrieved profiles from the raw data at the same date and time as presented in Fig. S1.

The single-half-way profile retrieved from i) shows larger fluctuations than the averaged profile. However, in order to eliminate and filter out short-range fluctuations, both aerosol backscatter profiles are smoothed in the next step.

3) Smoothening the vertical profile

A moving average window, using five data points (three data points under stable conditions), is used to filter the large fluctuations in the profiles (Fig. S3).
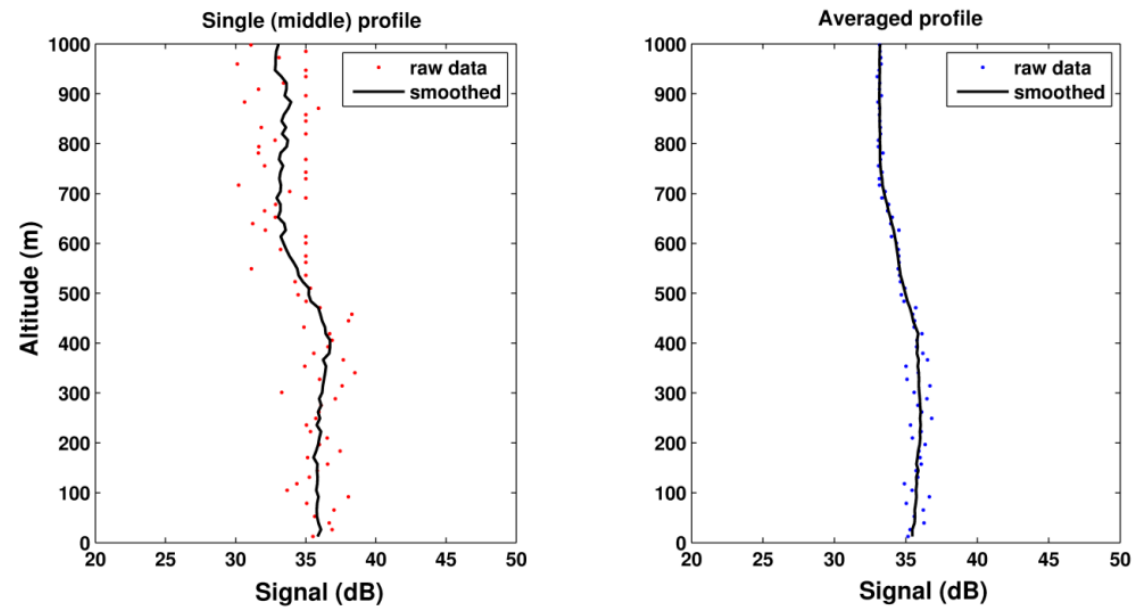

Figure S3: Smoothing the profiles of the backscatter signal (dB) using the moving-average method taking eight data-points average in the smoothing, for both, single (left) and averaged (right) profiles. Note that the profiles are the same as presented in Fig. S2, here divided into two panels.

4) Calculating the derivative of the backscatter (vertical)

The smoothed profiles of the raw backscatter signal indicate the layer of the maximal gradient. In our example, the maximum gradient height is between 400 and $600 \mathrm{~m}$. To calculate more precisely the location of the maximal gradient, we calculate the vertical derivatives of the signal elements in the smoothed profile array. More specifically, we take the derivate of every fifth element in the array, with respect to the previous fifth element, rather than taking the derivative of every second element along the array. This gives us a profile of the derivatives (vertical) of the smoothed backscatter profile with peaks at half of the distance between five elements within the array (Fig. S4a). We do this in order to prevent erroneous maximal gradients from the strong and sharp local differences (gradients) in the data. 
5) Finding the height of the maximal gradient (negative) in the smoothed profile

The height of the largest negative gradient is the height of the boundary layer for the corresponding profile (at that moment in time). Since we do not consider the positive gradients, we set them equal to zero (Fig. S4b). We then find the negative maximum in the derivative profile (Fig. S4b), and the height at which this maximum occurs (BL-height). Finally, in order to check if the height of the maximal gradient is consistent with the negative slope in the smoothed backscatter profile, we plot the line of the BL-height and visualize where it crosses the profile (Fig. S4c). The latter is visual confirmation (check) of the found BL-height.

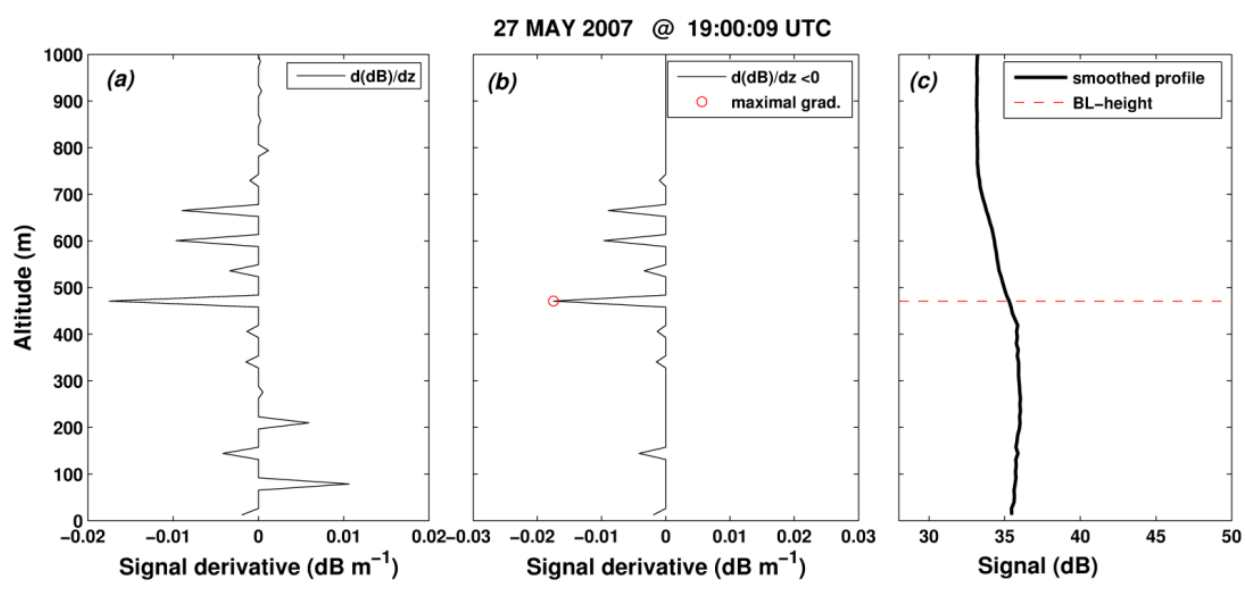

Figure S4: (a) Profile of the vertical derivative of the smoothed backscatter data; (b) the maximal negative gradient; (c) the boundary-layer height based on the smoothed profile for 27 May (19:00:09 UTC or 12:00:09 LT).

6) Plotting the heights of the maximal gradients (BL-height) in time (evolution)

In order to find the temporal evolution of the BL-height, we repeat the above procedure for all the lidar-scans in time. Here, we present the diurnal evolution of the BL-height for the $27^{\text {th }}$ of May 2007 (147th Julian day) (Fig. S5). We take this example because it was characterised by southerly winds, ensuring the largest fetch above the CHATS orchard.

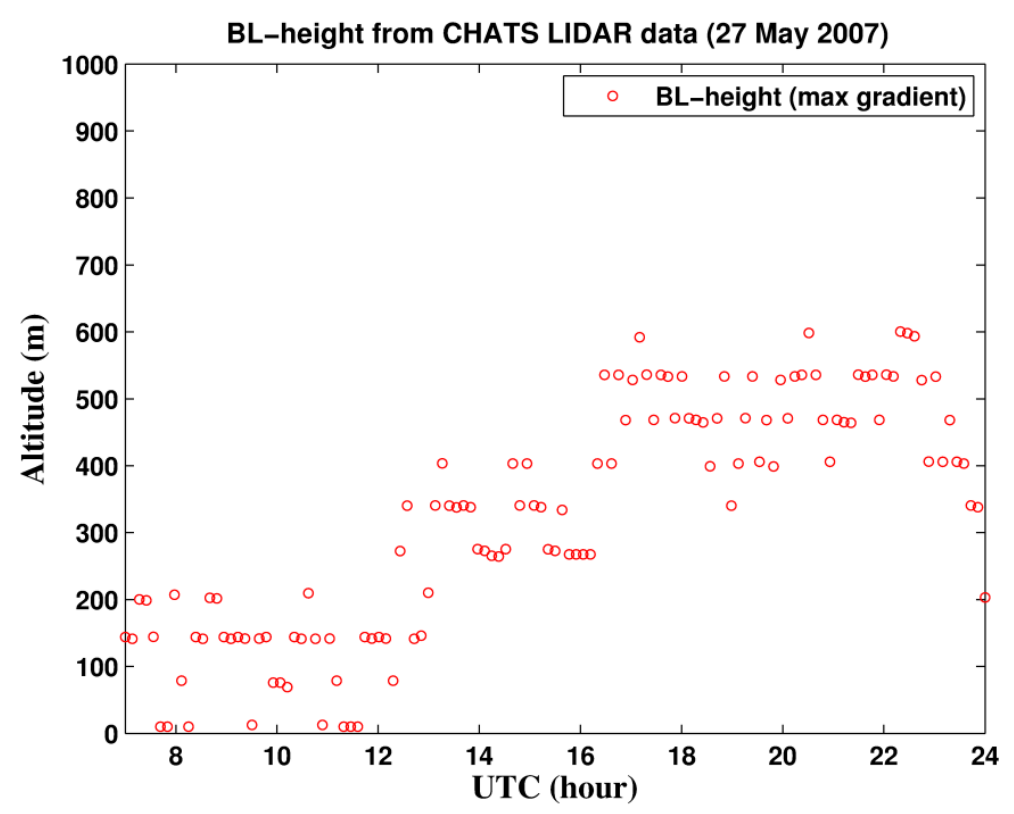

Figure S5: Temporal evolution of the boundary-layer height calculated from the CHATS LIDAR data for 27 May 2007.

Note that this method is valid for standard (typical) profiles of the backscatter. It is sensitive to atypical profiles (e.g. Fig B5), disrupted by advection effects that bring layers of aerosols with different characteristics, or for cases with a strong residual layer 
(e.g. morning-noon transition). Figure S5 shows ts an example in which the method described above has limitations. The left panel in Fig. S5 shows the aerosol concentration in distance and height. There are two heights at which the layers exhibit a sharp negative slope. The first is within the first $100 \mathrm{~m}$ and the second is between 300 and $400 \mathrm{~m}$. Since from this profile it is difficult to conclude where the BL-height should be, the method deals with this problem by choosing one of the two large maxima, which may not represent the true BL-height. This is illustrated in Fig. S6 (right panel); between 13:00 - 15:00 UTC (06:00 - 08:00 Local Time) we evaluated very high and very low values for the BL-height. In this case, the aerosol backscatter does not exhibit a regular profile, so the method to calculate the BL-height can lead to erroneous results. One possible solution would be to take the average height of both maxima, including the large fluctuation in the calculation as uncertainty (not shown).
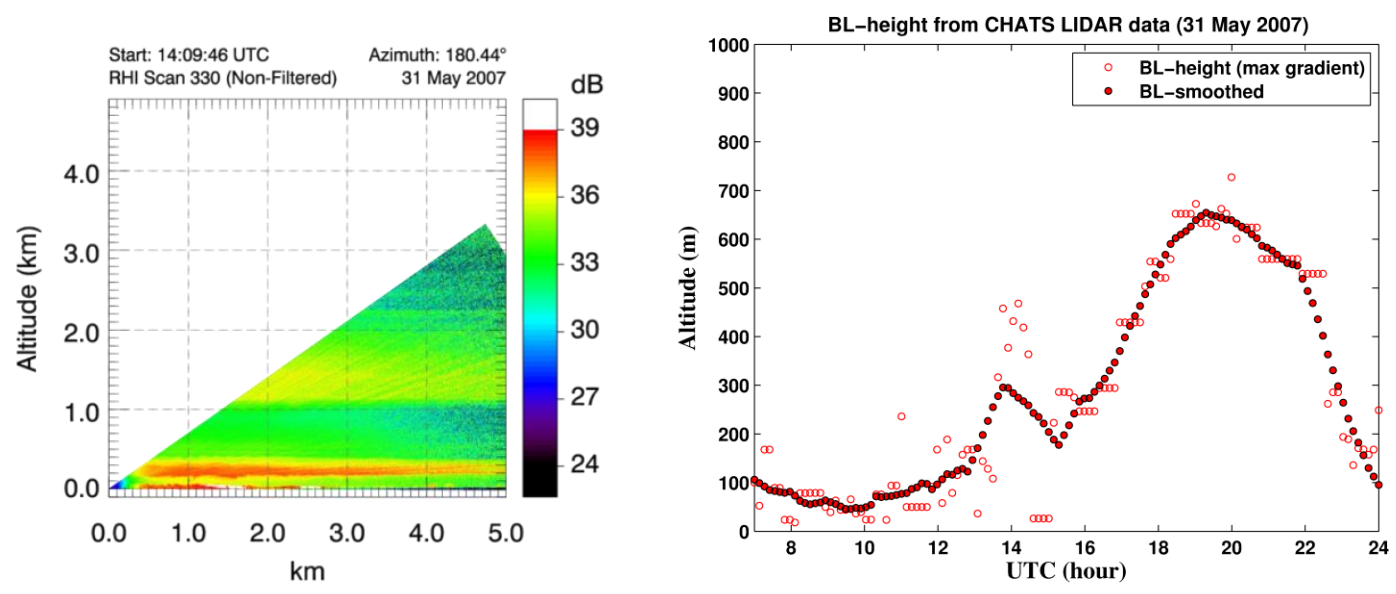

Figure S6: Similar as Fig. S1 and Fig. S5, but for 31 May 2007. Poorly represented transition period between 13:00 UTC (06:00 LT) and 15:00 UTC (08:00 LT). Note that the sunrise is around 13:30 UTC (06:30 LT).

\section{References}

Endlich, R., Ludwig, F., and Uthe, E.: An automatic method for determining the mixing depth from lidar observations, Atmos Environ, 13, 1051-1056, 1979.

Patton, E. G., Horst, T. W., Sullivan, P. P., Lenschow, D. H., Oncley, S. P., Brown, W. O. J., Burns, S. P., Guenther, A. B., Held, A., Karl, T., Mayor, S. D., Rizzo, L. V., Spuler, S. M., Sun, J., Turnipseed, A. A., Allwine, E. J., Edburg, S. L., Lamb, B. K., Avissar, R., Calhoun, R. J., Kleissl, J., Massman, W. J., Paw U, K. T., and Weil, J. C.: The Canopy Horizontal Array Turbulence Study, Bull Amer Meteor Soc, 92, 593-611, 2011. 